\title{
Aerodynamic performances and vehicle dynamic response of high-speed trains passing each other
}

\author{
Yinghui ZHAO, Jiye ZHANG*, Tian LI, Weihua ZHANG \\ Traction Power State Key Laboratory, Southwest Jiaotong University, Chengdu 610031, China
}

\begin{abstract}
Based on the aerodynamics and vehicle dynamics, the aerodynamic performances and vehicle dynamic characteristics of two high-speed trains passing each other on the ground, embankment and bridge are studied. Firstly, a train aerodynamic model and a vehicle dynamic model are established. Through the simulation of the two models, the pressure waves, aerodynamic forces, and vehicle dynamic responses are obtained. Then, the pressure waves and aerodynamic forces on different foundations are compared. The results show that the variation trends of pressure wave and aerodynamic forces of trains passing each other on different foundations are almost similar. The peak-to-peak differences in pressure wave and aerodynamic force are below $4 \%$ and $3 \%$ in three cases in open air. Besides, the differences of security indexes, including coefficient of derailment, wheel unloading rate, the wheelset lateral force, and the wheelrail vertical force, are below $2 \%$ among the three cases; the differences of comfort indexes, including the lateral acceleration and the vertical acceleration, are also below $2 \%$. It is concluded that the dynamic performances of trains passing each other are influenced little by different foundations in open air.
\end{abstract}

Key words: trains passing each other; foundations; pressure wave; aerodynamics; vehicle dynamics

(C) 2012 JMT. All rights reserved.

\section{Introduction}

$\mathrm{H}$ igh-speed train can induce strong disturbance to the surrounding air. When two high-speed trains are passing each other in open air, the disturbance will be aggravated, which would induce very large transient pressure fluctuations [1-3]. The pressure wave will lead to the deformation and lateral movement of car body, even the destruction of the window glass, and then influence the running security of the trains and the passenger comfort [4-7]. Tian et al. [8] studied the behaviors of trains passing each other at different speed levels and different line spacings. The research proved that the amplitudes of pressure wave of trains passing each other are proportional to the train speed, and that the relationship between pressure wave and line spacing is negatively exponential. Aiming at the computational efficiency, Ref. [9] proposed a method with only a half of the computational domain to the simulation problems of trains passing each other at the same speed. Because only a half of the computational domain is required, and the dynamic mesh technique is avoided, the computational efficiency is greatly improved. In addition, Khier

\footnotetext{
Received Jan. 5, 2012; revision accepted Feb. 28, 2012

*Corresponding author. Tel.: +86-28-86466040

E-mail: jyzhang@home.swjtu.edu.cn (J.Y.ZHANG)

(C) 2012 JMT. All rights reserved

doi: 10.3969/j.issn.2095-087X.2012.01.006
}

et al. [10] studied the flow structure around trains under side wind condition by simulation and obtained the aerodynamic performances of train running under side wind condition.

Two trains passing each other may happen on the ground, embankment, and bridge in open air. However, few researchers studied the effects of different foundations on the train aerodynamics and vehicle dynamics when trains passing each other. In this paper, we investigate the influences of different open foundations (ground, embankment and bridge) on the dynamic behavior of trains passing each other. The aerodynamic performances and vehicle dynamic responses of trains are simulated with the commercial software Fluent and Simpack. The pressure waves, aerodynamic forces and vehicle dynamic responses are analyzed on different foundations in open air.

\section{Aerodynamic model building}

\subsection{Governing equation of fluid dynamics}

When the two trains are passing each other at the speed of $380 \mathrm{~km} / \mathrm{h}$, the air flow around the trains is compressible, unsteady and turbulent in nature with high Reynolds number. On the basis of the standard $\kappa-\varepsilon$ two-equation, the equations of the flow around trains are written as [11]: 


$$
\begin{aligned}
& \frac{\mathrm{d}}{\mathrm{d} t} \int_{V} \rho \phi \mathrm{d} V+\int_{s}\left[\rho \phi\left(U-U_{g}\right)-\Gamma_{\phi} \operatorname{grad} \phi\right] \mathrm{d} S= \\
& \quad \int_{V} S_{\phi} \mathrm{d} V,
\end{aligned}
$$

where $\phi$ is flow flux, t is time, $\rho$ is density of fluid, $S$ is the area of control-volume interface, $V$ is volume, $U$ is the velocity of fluid, $U_{g}$ is the velocity of controlvolume interface, $\Gamma_{\phi}$ is the diffuse coefficient, and $S_{\phi}$ is the source term.

Ideal gas state equation is described as follows [12]:

$$
p=\rho R T,
$$

where $p$ is pressure, $\rho$ is density, $R$ is gas constant, and $T$ is temperature.

Finite volume method is used to solve Eqs. (1) and (2), and the second-order upwind difference formula is selected as the discrete format. Based on Eqs. (1) and (2), the pressure wave and aerodynamic forces of highspeed train are obtained by the simulation of the train aerodynamic model.

\subsection{Geometry models}

In order to study the behaviors of trains passing each other on different foundations (ground, embankment, and bridge), a certain high-speed train is chosen as the research object in this paper. The geometry models include train model, ground model, embankment model and bridge model. This paper studies the models of three-car marshalling and eight-car marshalling. The three-car marshalling model [13] includes one head car, one middle car, and one tail car. While the eight-car marshalling model includes one head car, six middle cars and one tail car. The head car and tail car have the same car appearance in both the cases. Moreover, in order to control the number of grids, the train is simplified as a $3 \mathrm{D}$ geometry with smooth surfaces, without considering the detailed features, such as pantographs, bogies and door handles, as shown in Fig. 1.
In reality, the structures of foundation include rails, sleepers, and so on. The sizes of those detailed structures are smaller than those of the whole track bed or the train. If considering all these details, the amount of calculation will be very enormous. Thus, the whole foundations are simplified as a 3D geometry with smooth surfaces, ignoring sleepers, track bed, and the detailed features such as bridge piers (Fig. 2).

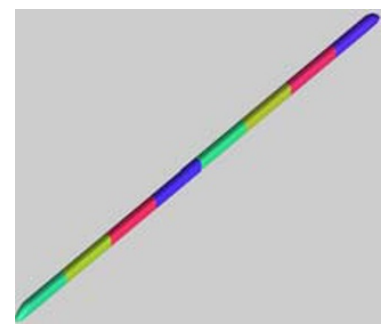

(a) Eight-car marshalling

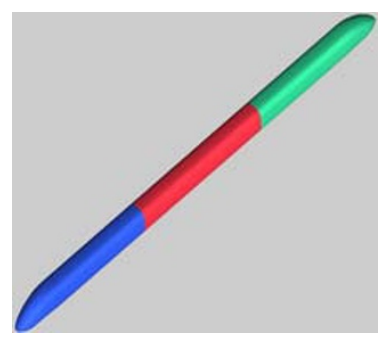

(b) Three-car marshalling
Fig. 1 Simplified train model (a) Ground

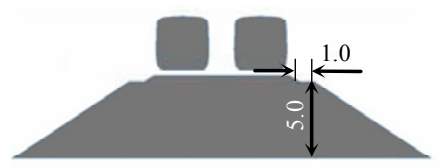

(b) Embankment

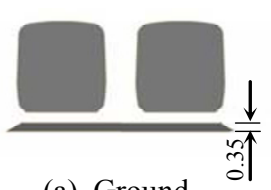

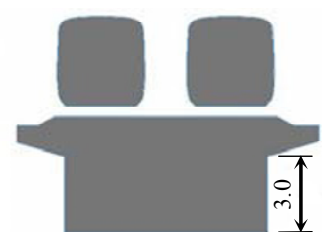

(c) Bridge

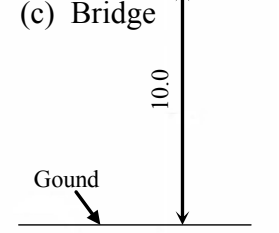

Fig. 2 Simplified model of foundations (unit: $\mathrm{m}$ )

\section{Verification of computational models}

In order to monitor the pressure wave of the train surface in the whole process of trains passing each other, some monitoring points are fixed on the train aerodynamic model (head car, middle car and tail car) in simulation, as shown in Fig. 3.

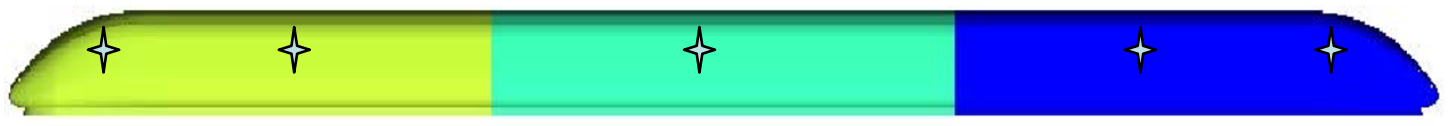

Fig. 3 Monitoring points on the window and door

The pressure waves of trains passing each other obtained from experimental [14] and simulation are shown in Fig. 4 under the same conditions (both are eight-car marshalling with the same speed and foundation environment). The peak-values and variation trend of the simulation results are almost consistent with the experi- mental results. Therefore, the calculation method of eight-car marshalling model is feasible.

The pressure wave and head-car side force $F_{x}$ of three-car marshalling model and eight-car marshalling model are shown in Fig. 5. By comparing the pressure wave and aerodynamic force of two models, it can be 
seen that the variation trends of two models are almost consistent, and the difference in peak values of two models is about $3 \%$.

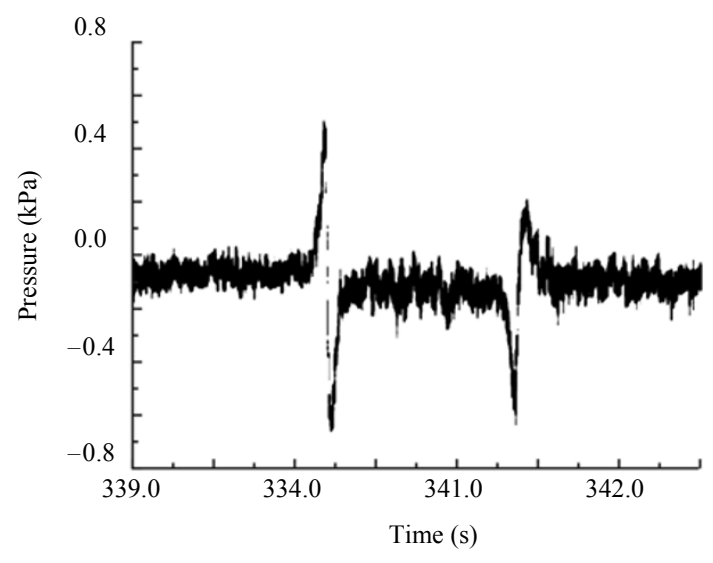

(a) Experiment results [14]
The pressure waves and aerodynamic forces of middle car and tail car have the similar patterns. As a result, using the three-car marshalling model to simulate the problem will be more economic.

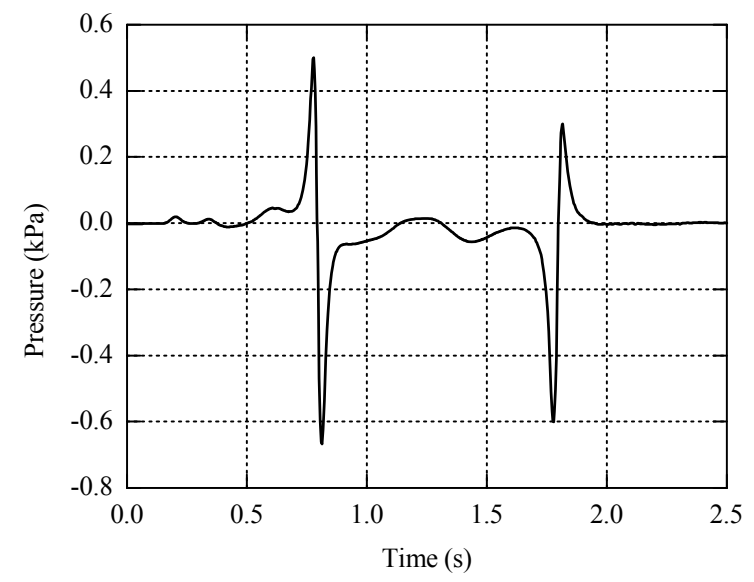

(b) Simulation results

Fig. 4 Pressure waves comparison between real car experiment and numerical calculation

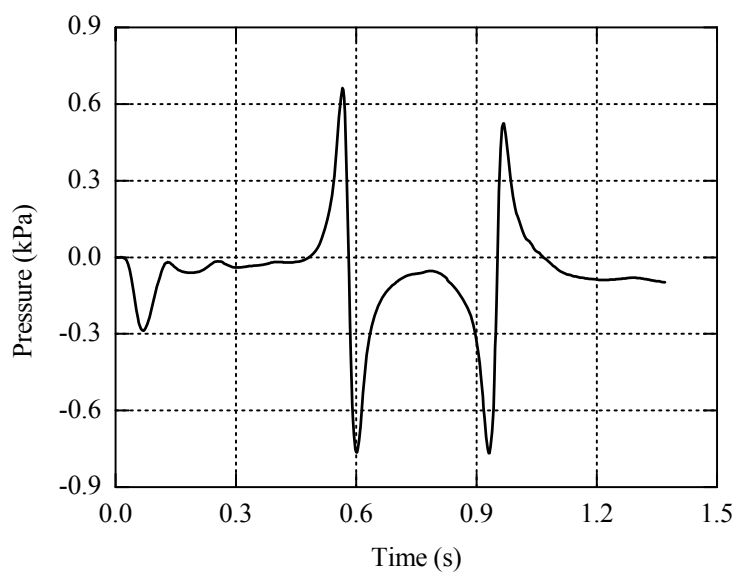

(a) Pressure wave of three-car marshalling

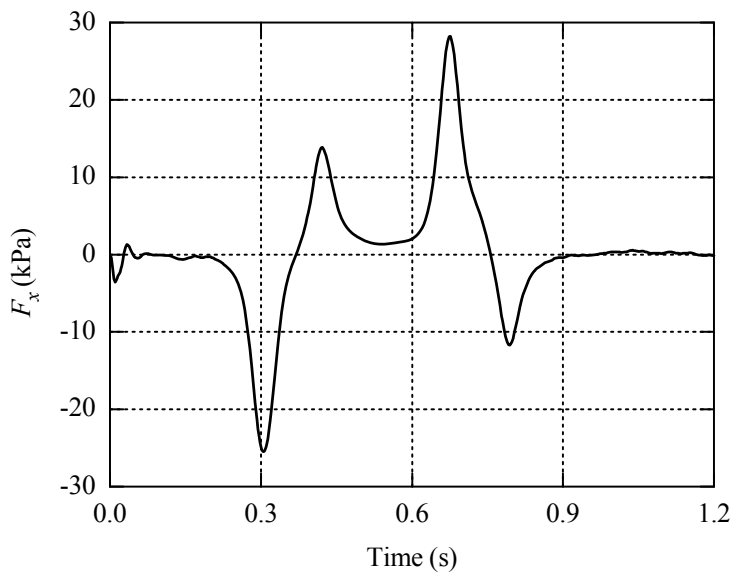

(c) Head-car side force of three-car marshalling

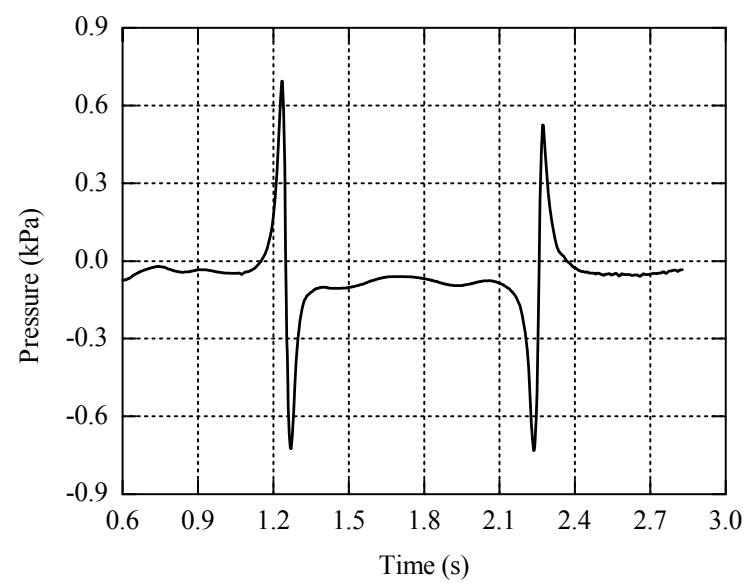

(b) Pressure wave of eight-car marshalling

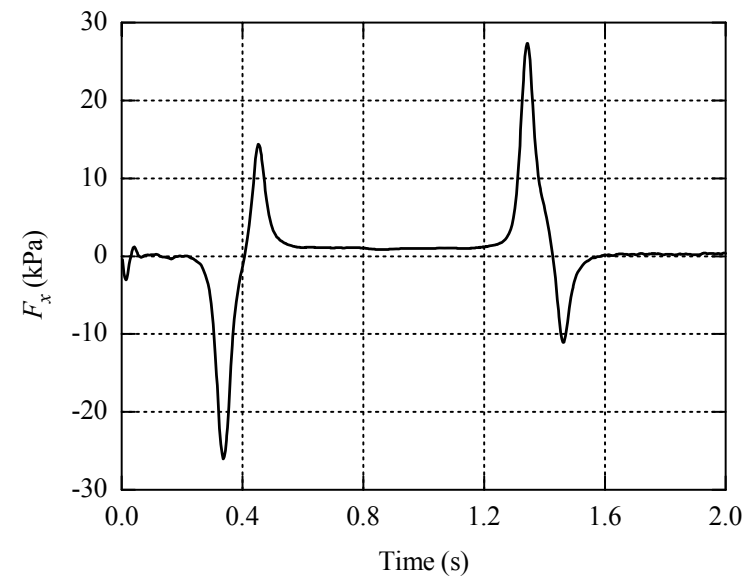

(d) Head-car side force of eight-car marshalling

Fig. 5 Comparison of three-car marshalling and eight-car marshalling 


\section{Analysis of simulation results of train aerodynamic performances}

In the simulation, six cases are simulated to study the aerodynamic performances of the trains passing each other on the ground, embankment, and bridge. The speed of train is $380 \mathrm{~km} / \mathrm{h}$. The height of embankment is $5.0 \mathrm{~m}$ and $7.0 \mathrm{~m}$, and the height of bridge is $10.0 \mathrm{~m}$, $12.0 \mathrm{~m}$ and $15.0 \mathrm{~m}$. The track spacing is $5.0 \mathrm{~m}$ and the height of track bed is $0.35 \mathrm{~m}$.

\subsection{Pressure wave of train passing each other}

The pressure waves of monitoring points on head car, middle car and tail car when trains passing each other on the ground, embankment and bridge at the speed of $380 \mathrm{~km} / \mathrm{h}$ are shown in Fig. 6 . We can see that the pressure waves of the corresponding monitoring points have a similar pattern. When the head car of one train is passing the monitoring points of the other train, the monitoring point pressure will increase and then drop suddenly; when the tail car is passing the monitoring point, the pressure wave has the opposite regular pattern.

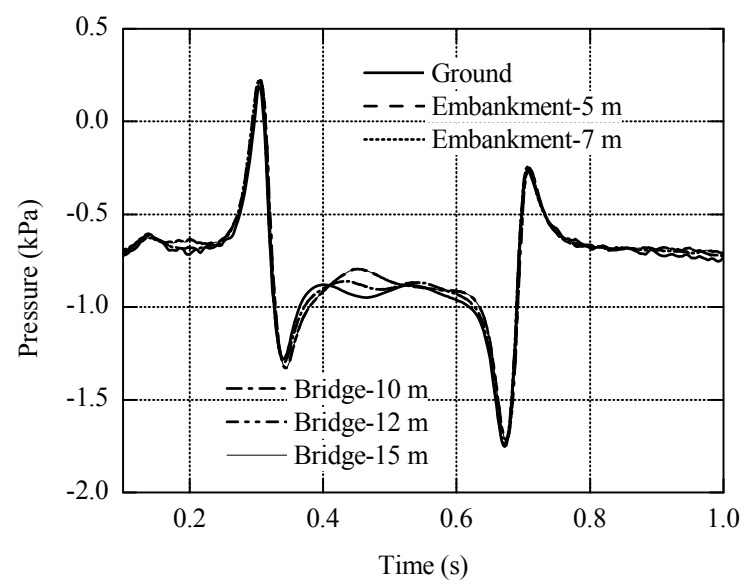

(a) Door of the head car

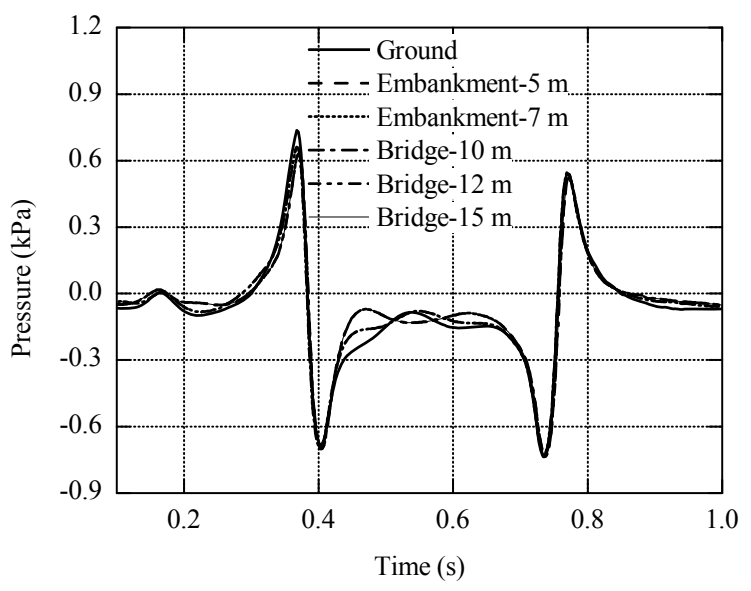

(b) Window of the head car

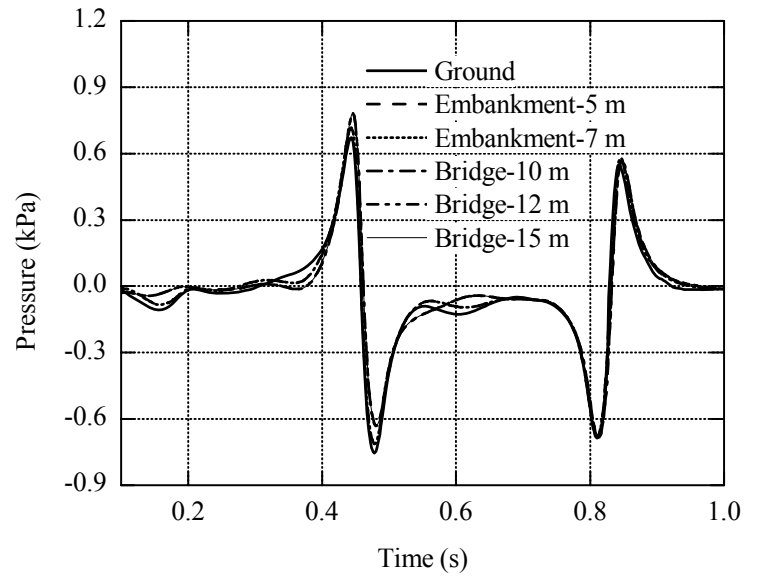

(c) Window of the middle car

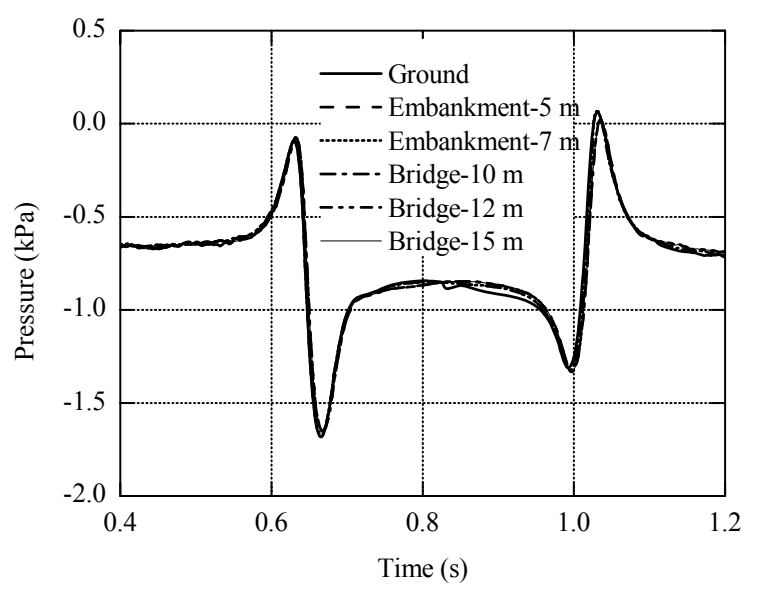

(d) Door of the tail car

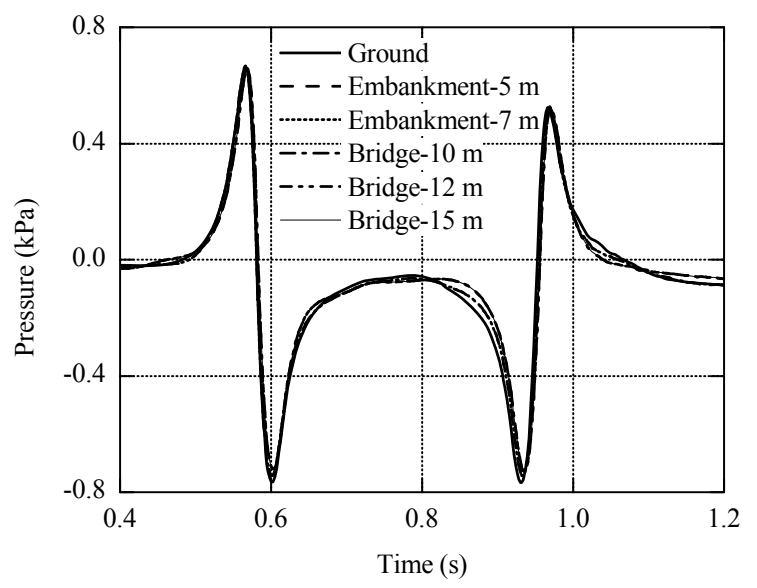

(e) Window of the tail car

Fig. 6 Pressure waves of different foundations on open line

Comparison of the peak-to-peak values of pressure waves for trains passing each other on the ground, embankment, and bridge are shown in Fig. 7. It is revealed that the peak-to-peak value on bridge is relatively smaller than on the embankment and ground. The differences of peak-to-peak values of all monitoring points are below $4 \%$, most of which are about $2 \%$. 


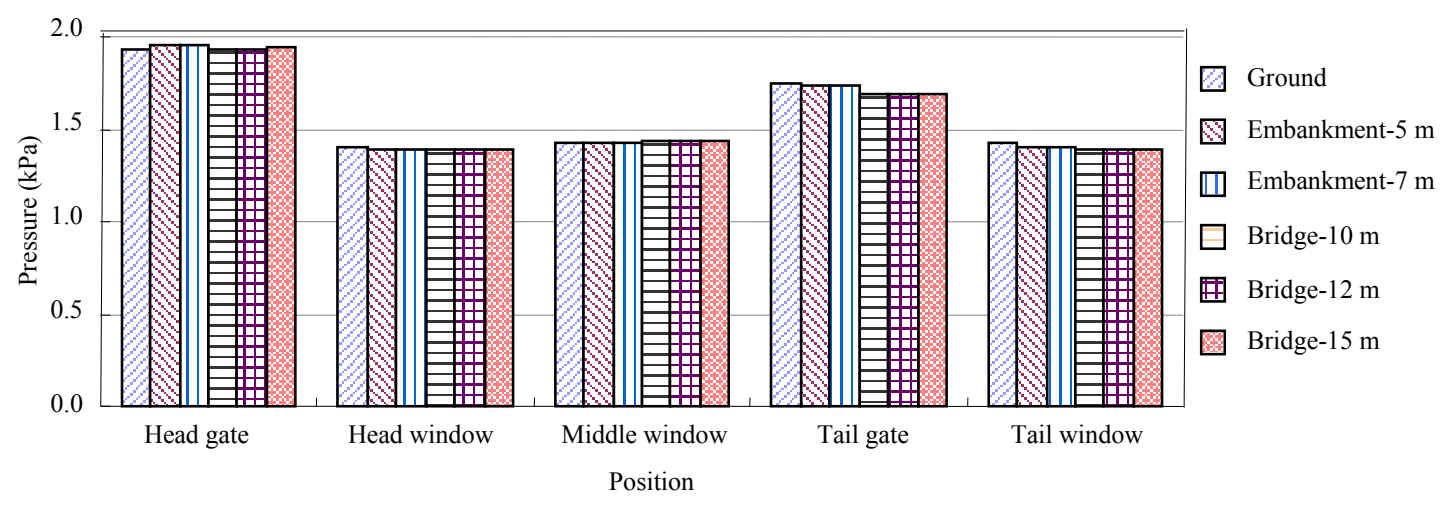

Fig. 7 Peak-to-peak values of pressure waves on different foundations

\subsection{Aerodynamic forces of trains passing each other}

The computation of aerodynamic force and moment is based on the coordinate in Fig. 8, side force $F_{x}$, lift force $F_{y}$, yaw moment $M_{y}$, and roll moment $M_{z}$. The aerodynamic performance of train 1 is chosen as the research object in this paper. The $F_{x}, F_{y}, M_{y}$, and $M_{z}$ of head car passing by at the speed of $380 \mathrm{~km} / \mathrm{h}$ are shown in Fig. 9. We can see that $F_{x}, F_{y}, M_{y}$, and $M_{z}$ on different foundations have the similar fluctuation pattern. The differences of peak-to-peak values of all aerodynamic forces are below $3 \%$, most of which are about $2 \%$.

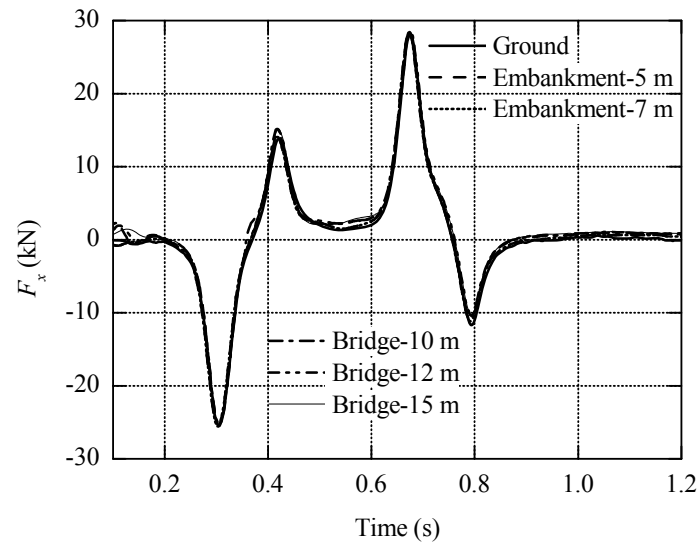

(a) Side force $F_{x}$

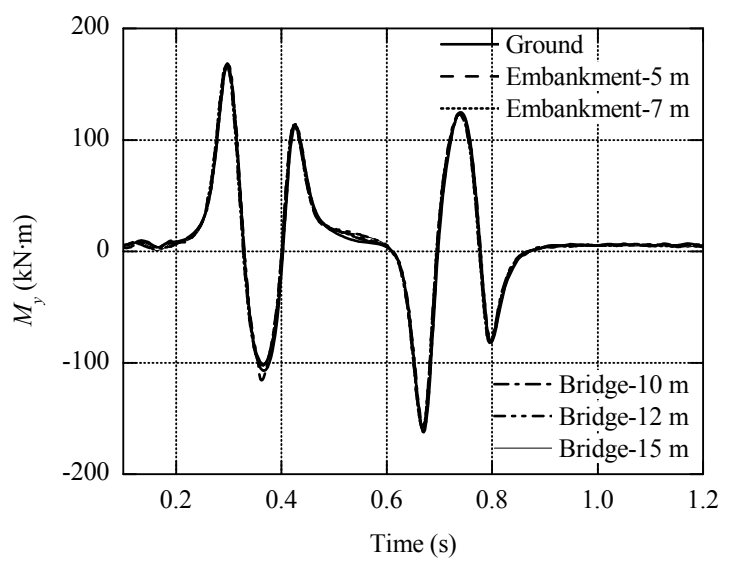

(c) Yaw moment $M_{y}$

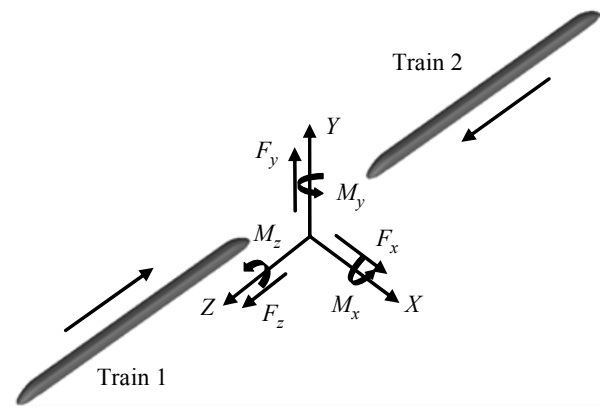

Fig. 8 Computation coordinate

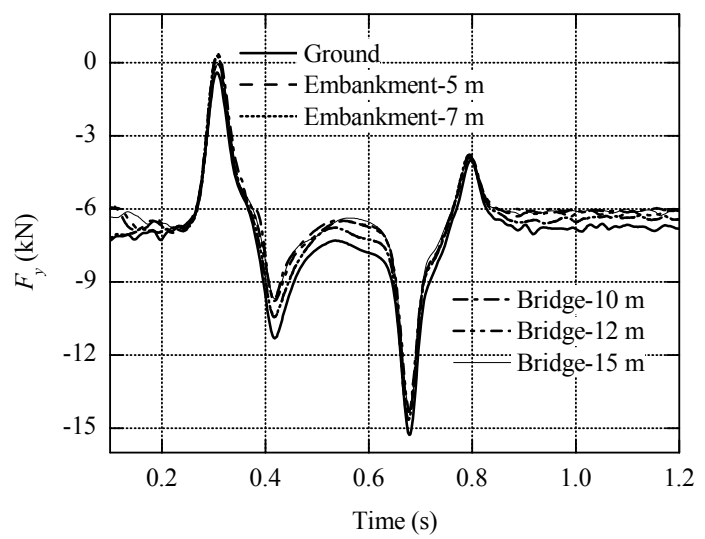

(b) Lift force $F_{y}$

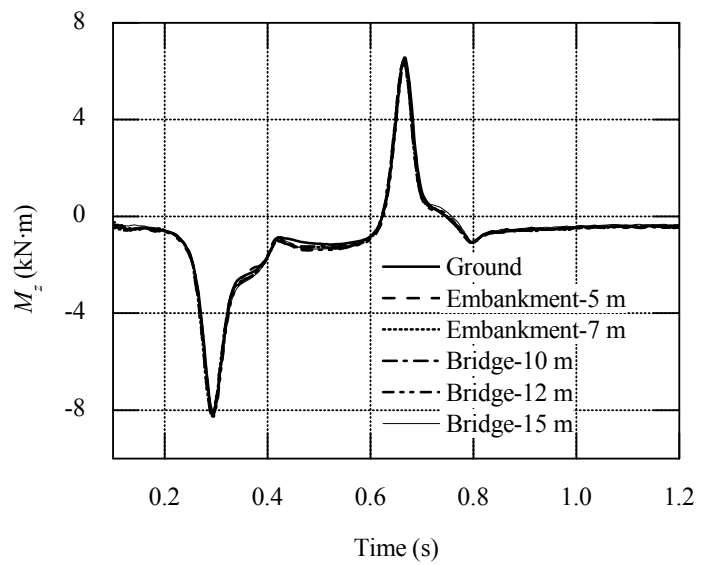

(d) Roll moment $M_{z}$

Fig. 9 Aerodynamic forces of the head car on different foundations 


\section{Vehicle dynamic model building}

In the dynamic modeling of high-speed trains, the suspension system and the vibration damper system are simplified to spring-damper force elements [15]. In this paper, we consider that the wheel-rail contact, wheel-rail force, yaw damper, lateral stop and the connection of spring and damper in series or in parallel are all geometrically linear in the vehicle dynamic model. The vehicle dynamic model chooses LMA and T60 as the wheel profile of the train and the rail respectively. The distance between backs of the wheel flanges is Chinese standard $1353 \mathrm{~mm}$, and the vehicle dynamic model chooses the measured track spectrum of Beijing-Tianjin high-speed railway as the track irregularity.

The equations of the train system dynamics are written as [16]:

$$
\boldsymbol{M} \ddot{\boldsymbol{X}}+\boldsymbol{C} \dot{\boldsymbol{X}}+\boldsymbol{K} \boldsymbol{X}=\boldsymbol{F}_{r}+\boldsymbol{F}_{a},
$$

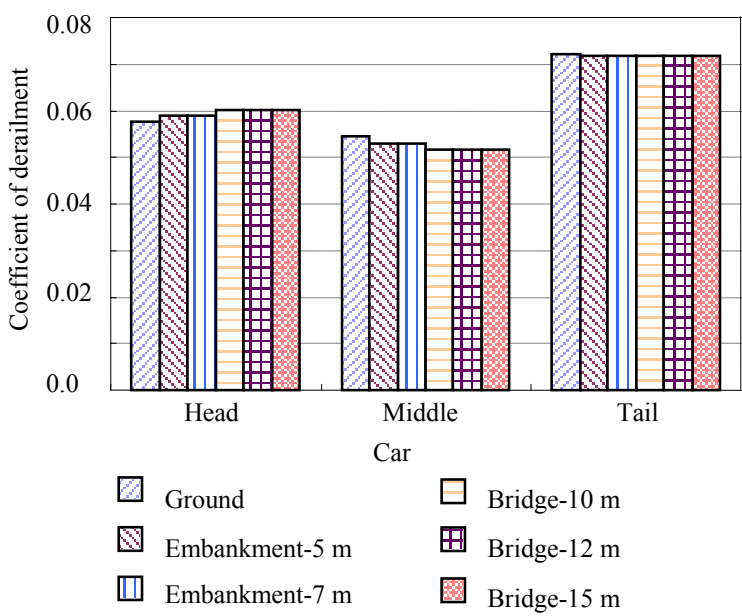

(a) Coefficient of derailment

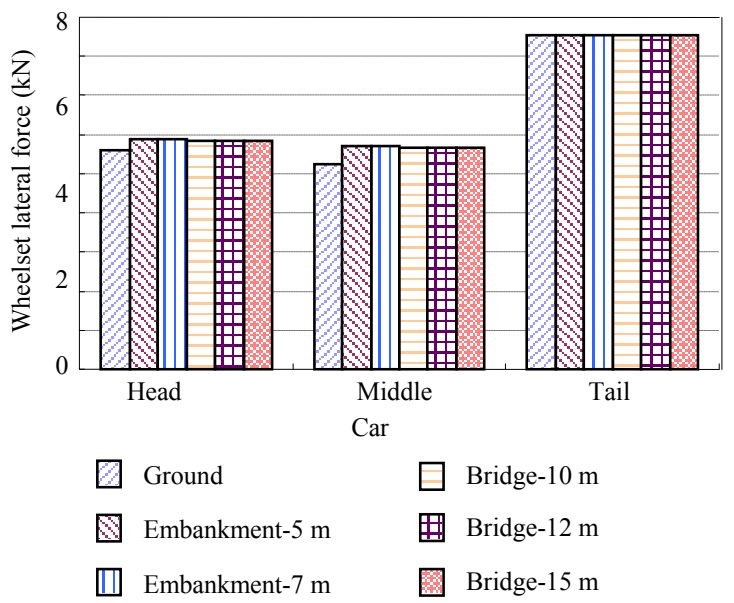

(c) Wheelset lateral force $(\mathrm{kN})$ where $\boldsymbol{M}, \boldsymbol{C}$, and $\boldsymbol{K}$ are the mass, damp and stiffness matrixes, respectively; $\boldsymbol{X}, \dot{\boldsymbol{X}}$, and $\ddot{\boldsymbol{X}}$ are the generalized displacement, velocity and acceleration vectors of the systems, respectively; $\boldsymbol{F}_{x}$ is the generalized load vector of the rail excitation; and $\boldsymbol{F}_{a}$ is the generalized load vector of the aerodynamics forces load. Based on Eq. (3), the vehicle dynamic responses (the coefficient of derailment, wheel unloading rate, the wheelset lateral force the wheel-rail vertical force, lateral acceleration and vertical acceleration) are obtained by the simulation of the vehicle dynamic model.

The coefficient of derailment, wheel unloading rate, the wheelset lateral force and the wheel-rail vertical force are used in the evaluation of running security of high-speed trains in this paper. The indexes should below these limitations: the coefficient of derailment $(Q / P)_{\lim }=0.80$, wheel unloading rate $(\Delta P / \bar{P})_{\lim }=0.80$, wheelset lateral force $H_{\mathrm{lim}}=10+P_{0} / 3,\left(P_{0}\right.$ is the axle load $)$

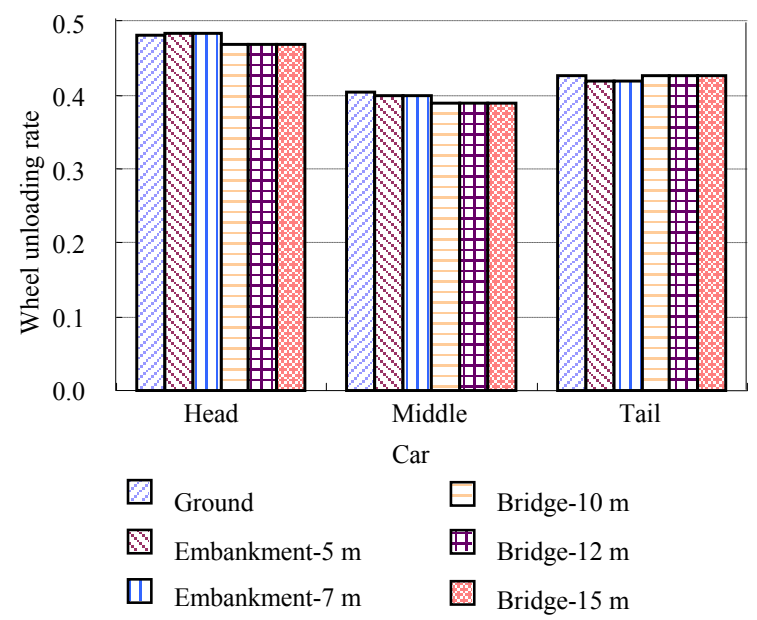

(b) Wheel unloading rate

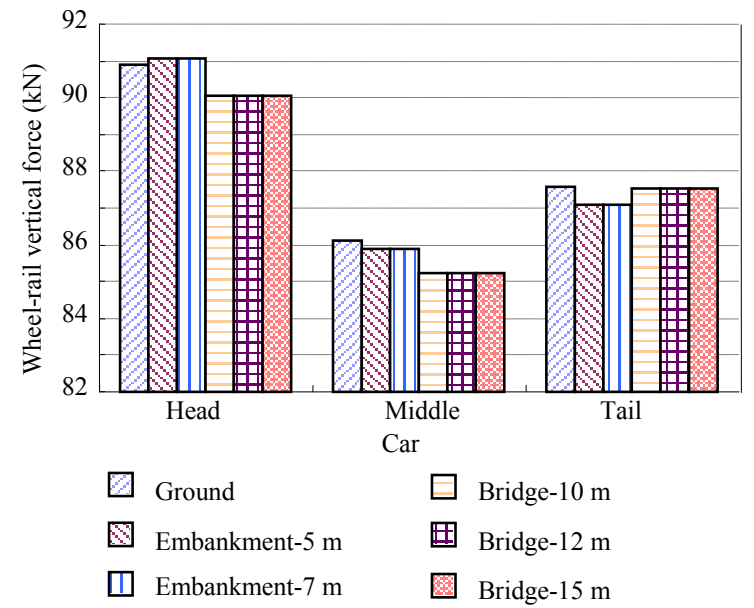

(d) Wheel-rail vertical force $(\mathrm{kN})$

Fig. 10 Safety index 
and the wheel-rail vertical force $P_{\text {lim }}=170 \mathrm{kN}$. In addition, lateral acceleration and vertical acceleration are used as the evaluation of passenger comfort of highspeed trains in this paper. The indexes should below these limitations: lateral acceleration $\left(a_{y}\right)_{\lim }=2.5 \mathrm{~m} / \mathrm{s}^{2}$, vertical acceleration $\left(a_{z}\right)_{\lim }=2.5 \mathrm{~m} / \mathrm{s}^{2}$.

\section{Analysis of simulation results of vehicle dynamic responses}

The indexes of security (coefficient of derailment,

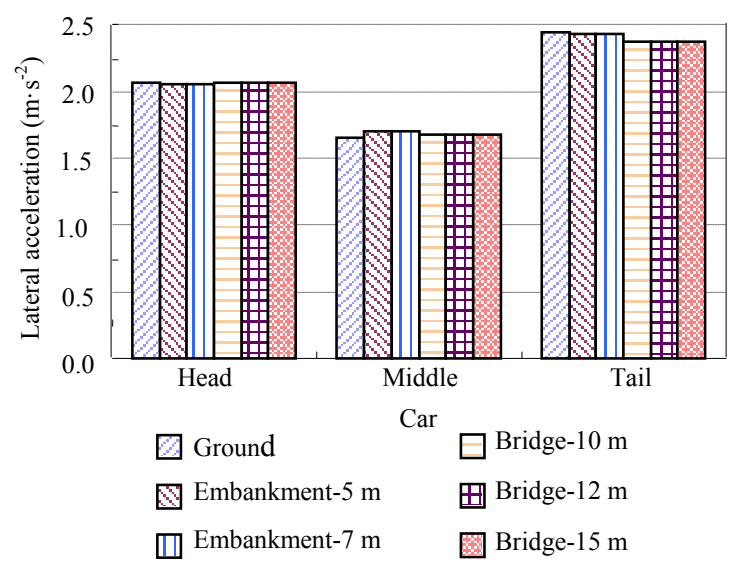

(a) Lateral acceleration $\left(\mathrm{m} \cdot \mathrm{s}^{-2}\right)$ wheel unloading rate, the wheelset lateral force and the wheel-rail vertical force) of high-speed trains passing each other on the ground, embankment and bridge at the speed of $380 \mathrm{~km} / \mathrm{h}$ are shown in Fig. 10. We can see that the differences of all the security indexes are below $2 \%$.

The comfort indexes (the lateral acceleration and the vertical acceleration) of high-speed passing trains on the ground, embankment and bridge at the speed of $380 \mathrm{~km} / \mathrm{h}$ are shown in Fig. 11 . We can see that the differences in all the comfort indexes are below $2 \%$.

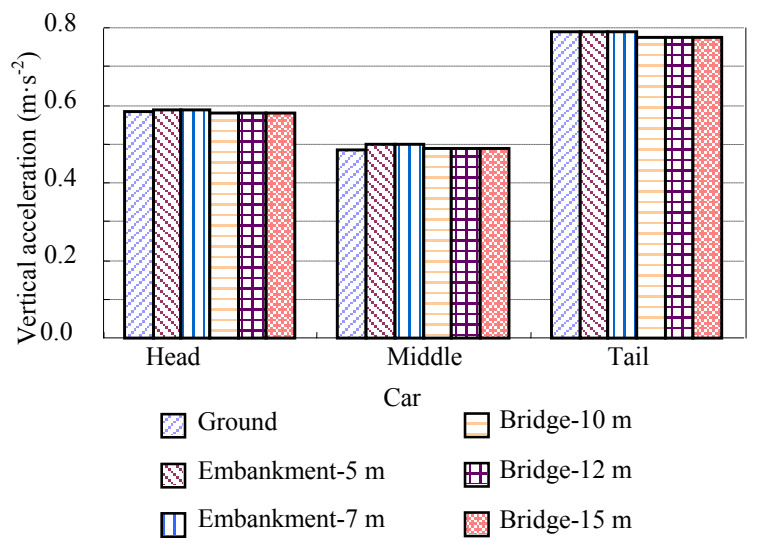

(b) Vertical acceleration $\left(\mathrm{m} \cdot \mathrm{s}^{-2}\right)$

Fig. 11 Comfort indexes

\section{Conclusion}

Based on the analysis of the results, the following conclusions can be drawn:

(1) When two trains are passing each other on the ground, embankment and bridge, the pressure waves of the corresponding monitoring points have the similar regular pattern at the same speed; the differences of the peak-to-peak values are below $4 \%$.

(2) The aerodynamic forces of the passing trains have the similar regular pattern at the same speed on the ground, embankment, and bridge; the differences of the peak-to-peak values are below 3\%.

(3) The differences in all the indexes of comfort and security of trains passing each other on the ground, embankment, and bridge are below $2 \%$.

(4) The train aerodynamics and vehicle dynamics of trains passing each other are influenced little by different foundations in open air.

\section{Acknowledgements}

The research was supported by the National Natural Science Foundations of China (Nos. 50823004 and
50821063) and the "Eleventh Five-year Plan" National Science and Technology Support Plan (No. 2009BAG12A01-C09).

\section{References}

[1] H.Q. Tian, Study development of train aerodynamics in China, Journal of Traffic and Transportation Engineering, 2006, 6(1): 1-9 (in Chinese).

[2] R.S. Raghunathnana, H.D. Kimb, T. Setoguchi, Aerodynamics of high-speed railway train, Progress in Aerospace Sciences, 2002. 38(6): 469-514.

[3] K. Fujii, T. Ogawa, Aerodynamics of high speed trains passing by each other, Computers Fluids, 1995, 24(8): 897-908.

[4] J. Hwang, T. Yoon, D. Lee, et al., Numerical study of unsteady flow field around high speed trains passing by each other, JSME International Journal, Series B, 2001, 44(3): 451-464.

[5] J.L. Liu, Computation of two passing-by high-speed trains by a relaxation overset-grid algorithm, International Journal for Numerical Methods in Fluids, 2004, 44: 1299-1315.

[6] H.Q. Tian, S. Yao, S.G. Yao, Influence of the air pressure pulse on car-body and side-windows of two meeting trains, China Railway Science, 2000, 21(4): 6-12 (in Chinese). 
[7] X.B. Li, J.Y. Zhang, W.H. Zhang, Simulation research on vibration caused by airflow while high-speed trains passing each other, Rolling Stock, 2009, 47(12): 9-12 (in Chinese).

[8] H.Q. Tian, P. Xu, X.F. Liang, et al., Correlation between pressure wave of train passing and running speed, China Railway Science, 2006, 27(6): 64-67 (in Chinese).

[9] X.L. Zhao, Z.X. Sun, A new method for numerical simulation of two trains passing by each other at the same speed, Journal of Hydrodynamics, 2010, 221(5): 697702.

[10] W. Khier, M. Beruer, F. Durst, Flow structure around trains under side wind condition: a numerical study, Computers \& Fluids, 2000, 29(2): 179-195.

[11] W.P. Li, Computational Fluid Dynamics, Wuhan: Huazhong University of Science and Technology Press, 2004: 49-58 (in Chinese).
[12] W.Y. Wu, Fluid Dynamics, Beijing: Peking University Press, 1998 (in Chinese).

[13] X.B. Li, Z. Yang, J.Y. Zhang, et al., Aerodynamics properties of high-speed train in strong wind, Journal of Traffic and Transportation Engineering, 2009, 9(2): 6673 (in Chinese).

[14] X.F. Liang, H.Q. Tian, Test research on crossing air pressure pulse of electric multiple unit, Social Science Journal for Central South University of Technology, 2002, 33(6): 621-624 (in Chinese).

[15] W.M. Zhai, Vehicle Track Coupling Dynamics, 2nd ed., Beijing: China Railway Publishing House, 2002 (in Chinese).

[16] T. Li, J.Y. Zhang, W.H. Zhang, An Improved algorithm for fluid-structure interaction of high-speed trains under crosswind, Journal of Modern Transportation, 2011, 19(2): 75-81. 